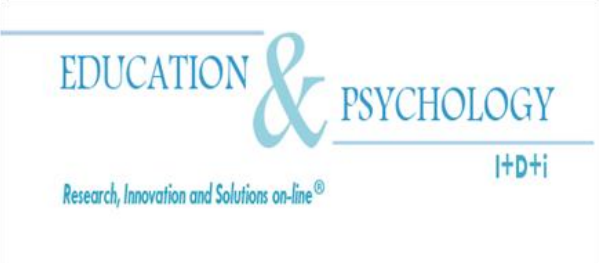

\title{
Estudio de las atribuciones hacia el maltrato entre iguales del alumnado agresor frente al no agresor, mediante el Cuestionario SCAN-Bullying
}

\section{Rosaura Gutiérrez $^{1}$, Juan L. Benítez ${ }^{1}$, Carlos Machado ${ }^{2}$ y Fernando Justicia ${ }^{1}$}

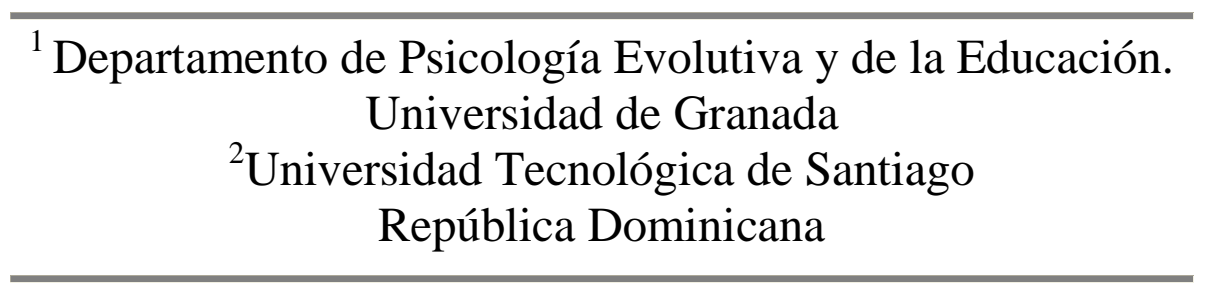

\section{República Dominicana y España}




\section{Resumen}

Introducción. El problema del maltrato entre iguales afecta directamente al clima de convivencia de un centro en general y al clima del aula en particular. Por esta razón, se hacen necesarias herramientas que permitan implementar intervenciones que promuevan la convivencia sana entre compañeros. En este sentido, el conocimiento de la percepción que el alumnado tiene hacia el maltrato entre iguales es relevante para el establecimiento de medidas adecuadas. Para tal fin, se pretende determinar la valoración del alumnado (agresor y no agresor) respecto a determinadas actitudes y acciones frente al maltrato entre iguales. Y en segundo lugar, conocer la relación entre las actitudes y las acciones más valoradas y el rol del alumnado.

Participantes. La muestra está formada por 97 estudiantes de $6^{\circ}$ y $7^{\circ}$ grado con edades comprendidas entre los 10-16 años $(\bar{X}=12.82 ; \mathrm{dt}=1.2)$ de los cuales el $51,5 \%$ son hombres y el $48,5 \%$ mujeres.

Instrumento. Para la realización del estudio se utilizan dos instrumentos. La Escala DIAS de agresión y victimización directa e indirecta para la identificación de roles entre el alumnado. Y el Cuestionario Scan-Bullying para el estudio de las atribuciones mentales del alumnado.

Resultados. Los resultados obtenidos indican un alto porcentaje de alumnado agresor entre los participantes siendo más elevada la tasa de agresores entre los hombres. Se encuentran diferencias estadísticas puntuales en relación a las atribuciones realizadas por agresores y no agresores respecto al maltrato entre iguales.

Discusión y conclusiones. Tanto los nominados agresores como no agresores consideran el maltrato como hechos intencionados, de maldad, reprobables, injustos e incorrectos. El alumnado agresor es consciente de la incorrección del maltrato y lo justifica sólo atendiendo a que es una forma de defenderse y no de actuar.

Palabras clave: Atribución cognitiva, agresores reactivos, agresores proactivos, maltrato entre iguales, convivencia. 


\title{
A study of mental attributions toward bullying in students ages 10 to 16, through the SCAN-Bullying Questionnaire
}

\begin{abstract}
Introduction. The phenomenon of bullying affects directly the school climate in general and the classroom climate. For this reason, it is necessary to create tools in order to implementate interventions for improving a right connivance among students. In this sense, the knowledge about students' perception about bullying is relevant for establishing appropriate interventions. Due to this aim, we pretend to determine the students' appreciation (aggressor and nonaggresor) towards certain attitudes and actions facing bullying. Second, we want to know the relation between the most valued attitudes and actions and the student's role.
\end{abstract}

Sample. 97 students from 10 to 16 years old $(\bar{X}=12.82 ; \mathrm{sd}=1.2)$ who are at 6 th and 7 th grade of education into the Brasilian educational system.

Instrument. For working out the study two instruments were used. The DIAS Scale of direct/indirect aggression and victimization for identifying roles among students. The ScanBullying Questionnaire for studying students' mental attributions.

Results. The results point out a high percentage of aggressors among participants and specially higher among males. We find some statistical differences related with the aggressors and non-agressors' attributions towards bullying.

Discussion and conclusion. For the nominees as aggressors, as well as for non-aggressor, the phenomenon of bullying is considered intentional, badness, blameworthy, unfair and improper. The aggressors are consciente about the incorrectness of bullying and they justify it only attending to a way of defense and not a way of acting.

Keywords: Cognitive attributions, reactive aggressors, proactive aggressors, bullying, connivance. 


\section{Introducción}

El maltrato entre iguales en los centros educativos ha sido objeto de estudio en cuanto a la definición, categorización, tasa de incidencia, factores etiológicos, etc. (Benítez y Justicia, 2006) y ha sido abordado desde diferentes enfoques en el área de investigación científica (Abramovay y Rua, 2002; Avilés, 2001; Collell y Escudé, 2003; Devine 2002; Fante 2005; Gonçalves y Sposito, 2002; Moos, Moos y Trickett, 1988; Ortega y Merchán, 1996; Thums, 2003). A pesar de las ricas contribuciones, con respecto al maltrato entre iguales, no ha sido fácil encontrar una definición consensuada. Sin embargo, a raíz de las contribuciones de Olweus (1993), se mantienen como elementos importantes en su definición: el carácter físico y/o verbal de la agresión; la intencionalidad; la frecuencia; la duración; y, el desequilibrio de poder entre agresor y víctima (Benítez y Justicia, 2006). Sin embargo, y en pos de una definición más completa y consensuada, es necesario incorporar la agresión relacional como otra característica del maltrato entre iguales que tiene lugar cuando suceden situaciones de exclusión o rechazo social (Del Barrio, Gutiérrez, Barrios, Van der Meulen y Granizo, 2005).

En cuanto a la incidencia del maltrato, es importante señalar que a pesar de la no homogeneidad de los instrumentos utilizados y de las características definitorias del constructo utilizadas por cada estudio, los resultados en varios países apuntan a la gravedad y existencia del problema. Desde los primeros estudios realizados por Olweus (1991) hasta los más recientes (Defensor del Pueblo, 2006; Garaigordobil y Oñederra, 2008; Ramírez y Justicia, 2006; Yuen Man Yan, 2007, Caurcel 2009) muestran tasas de agresión y victimización similares.

En relación a las conductas agresivas, varios autores distinguen entre la agresión reactiva y la agresión proactiva (Benítez y Justicia, 2006; Berkowitz 1963, 1993, Caurcel, 2009). La agresión reactiva está acompañada por rabia y se considera como reacción inmediata e impulsiva a una situación (real o percibida) de provocación, frustración o de amenaza. Deficiencias en el procesamiento de la información social pueden contribuir a desarrollar y mantener este patrón de conducta agresiva (Crick y Dodge 1996; Dodge y Coie 1987). Existe un modelo en cadena que describe los procesos internos entre el acto de provocación-frustraciónamenaza y la reacción agresiva reactiva: (1) codificación, (2) interpretación, (3) selección de un objetivo de acto de agresión, (4) generación de respuestas posibles, (5) selección de posibles respuestas, y (6) la acción (Crick y Dodge 1996; Dodge y Coie 1987). Los agresores re- 
activos presentan déficits en relación con la codificación y la interpretación de experiencias dolorosas y repetidas de victimización, que son almacenadas en la memoria a largo plazo, y cuando perciben algo que se asocia con estas experiencias, los recuerdos y las emociones correspondientes son activados causando frustración. Los agresores reactivos perciben hostilidades aún cuando en realidad no las hay necesariamente y como respuesta generan soluciones agresivas (Crick y Dodge, 1996). La agresión proactiva encaja en otro modelo que describe las cadenas de procesos personales basándose en el modelo del aprendizaje social (Bandura, 1973, 1983). Los humanos tienden a imitar modelos de conducta de otros (Turnbull y Patterson, 1999). Así, se tiende a adoptar conductas agresivas cuando se espera conseguir algo deseado. Para los agresores proactivos, los actos de agresión no son respuestas a alguna amenaza o frustración, sino que la agresión se usa como instrumento para lograr algo.

Por otro lado, es importante destacar el rol que, durante la adolescencia, juegan el proceso de la construcción de la identidad y la pertenencia a un grupo de referencia. Esto se debe a que muchos de los problemas disciplinares y conductuales se originan por pensamientos y constructos mentales relacionados con la idea de no ser querido y valorado por los compañeros. En este sentido, es importante investigar sobre las atribuciones mentales del alumnado con la finalidad de conocer sus percepciones y actitudes, para una vez conocidas, generar alternativas ante situaciones de maltrato, de forma que sean más proactivas e menos reactivas. El estudio de Benítez, Almeida y Justicia (2007) apunta que el acompañamiento y formación en los alumnos tiene efectos positivos, sobre todo, en lo que tiene que ver en la resolución de conflictos, la empatía, la disponibilidad de ayuda a los demás y el respeto a los demás. En la misma línea, Cowie (2000) y McLoughlin (2009) señalan que el alumnado manifiesta sentirse bien ayudando a otros, lo que aumenta la autoconfianza, mejora la autoestima y con ello, mejoran los constructos mentales de abordar situaciones generadoras de conflictos.

En relación con lo anterior, resulta de gran interés conocer las diferencias en cuanto a las atribuciones mentales del alumnado frente al maltrato entre iguales, y en especial, las del alumnado agresor para conocer cómo entiende, percibe y justifica el acto del maltrato (Avilés, 2006). Para tal fin existen varias aportaciones realizadas en la recogida de datos por medio de uso de cuestionarios de nominación de pares y otros instrumentos que sirven para diagnosticar la realidad de abuso y malos tratos, las posibles causas, las condiciones de riesgos a nivel evolutivo y ecológico (Jimerson, Swearer y Espelage, 2010; Phillips y Dewey, 2012). Por otra parte, y en los últimos años, se han dado a conocer otros métodos que procuran investigar los 
pensamientos, la apreciación que tienen los alumnos sobre actos de malos tratos y las diferentes cogniciones desde los roles asignados, agresor, victima, agresor-victima, observador (Almeida, Lisboa y Caurcel, 2007; Caurcel, 2009; Fernández, 2006; Smorti, 1994).

\section{Objetivos e hipótesis}

En relación a lo anterior, el presente estudio pretende por un lado detectar al alumnado agresor y no agresor, y sobre la identificación realizada:

- Conocer la valoración que los alumnos hacen de determinadas actitudes y acciones frente a una historia de maltrato de manera intencional, repetida, siendo la victima incapaz de defenderse por sí sola.

- Determinar la relación entre las valoraciones de actitudes y acciones por cada participante y el grado de agresión hacia sus pares que presentan.

Del mismo modo, se pretende ratificar la siguiente hipótesis:

HP1. El alumnado agresor, en las diferentes formas de agresión, presentará un perfil específico y diferente al alumnado no agresor en relación a las percepciones, sentimientos y actitudes frente al maltrato entre iguales.

\section{Método}

\section{Participantes}

La muestra (Tabla 1) está formada por estudiantes $(\mathrm{N}=97)$ de una escuela del municipio de Santarém (Pará, Brasil). Los participantes (51,5\% hombres y 48,5\% mujeres) pertenecen a cursos de $6^{\circ}$ y $7^{\circ}$ grado con edades comprendidas entre los 10-16 años $(\bar{X}=12,82$; $\mathrm{dt}=1,2)$. 
Tabla 1. Distribución de la muestra por sexo, edad y curso.

\begin{tabular}{ccc}
\hline Curso & Sexo & $\mathrm{N}$ \\
\hline $6^{\circ}$ & Chicas & $19(57 \%)$ \\
& Chicos & $14(43 \%)$ \\
$7^{\circ}$ & Chicas & $28(44 \%)$ \\
& Chicos & $36(56 \%)$ \\
\hline
\end{tabular}

\section{Instrumentos}

La Escala DIAS -Direct and Indirect Aggression Scales- (Björkqvist, Lagerspetz y Österman, 1992) consta de 48 ítems que hacen referencia a diferentes tipos de agresión directa e indirecta distribuidos en dos subescalas: una de agresión directa e indirecta (24 ítems) y otra de victimización directa e indirecta (24 ítems). Ambas subescalas se inscriben dentro de los métodos de nominación por pares en la que los participantes indican quién realiza/sufre las prácticas del maltrato y el nivel de frecuencia con el que lo hacen utilizando para ello una escala tipo Likert de cuatro niveles (1-raramente, 2-a veces, 3-bastante, 4-muy frecuentemente). El objetivo del cuestionario no es otro que identificar a agresores, víctimas y espectadores del maltrato (Justicia, Arco y Benítez, 2003). La validación de la consistencia interna de la versión portuguesa del instrumento presenta un valor de ,98 en su totalidad (Machado, 2002). De forma más específica, se obtuvieron los siguientes valores de consistencia interna: ,95 para victimización/agresión física, ,95 para victimización/agresión indirecta, y ,94 para la dimensión de agresión indirecta/victimización verbal (Machado, 2002).

Las escalas se tabulan sumando las puntuaciones obtenidas en la escala Likert de cada nominado. Cada nominador indica, para cada ítem, el nombre de los compañeros que a su juicio cometen actos agresivos y la frecuencia con la que suceden, pudiendo indicar tantos nombres como sean necesarios. En las casillas de los nominados, se introduce la frecuencia de nominación, es decir, el valor de nominación otorgado por los compañeros. Al final de cada nombre se obtiene un valor que será la resultante de sumar todas las puntuaciones obtenidas por el alumno en función de las respuestas dadas por los compañeros.

Las puntuaciones obtenidas sirven para determinar los índices de agresión de cada alumno. No obstante, son puntuaciones directas que posteriormente se ponderan para obtener una puntuación específica del índice. Para ello, se divide la puntuación total obtenida en los ítems entre el número total de ítems. El resultado se divide entre el número total de nominado- 
res, y se multiplica por 100 (Machado, 2002). Los valores obtenidos permiten calcular el promedio de este índice para todo el curso para posteriormente comparar los resultados a nivel individual y establecer si el índice de agresión está por encima o por debajo del promedio del curso. El alumnado con puntuaciones por encima del promedio del curso se clasifican como agresores, y el alumnado con puntuaciones por debajo del promedio del curso se establecen como no agresores.

El Cuestionario del SCAN-Bullying (Almeida y Caurcel, 2005; Del Barrio, Almeida, Van der Meulen, Barrios y Gutiérrez, 2003) -acrónimo inglés de Scripted-Cartoon-Narrativeestá diseñado para conocer los procesos cognitivos, las apreciaciones, las atribuciones mentales, los sentimientos, las emociones y las estrategias de afrontamiento que el individuo exhibe frente a una situación de maltrato. El instrumento es una aplicación narrativa compuesta por 15 láminas con viñetas (Figura 1), en versión masculina y femenina, que representan de manera secuencial la historia de un alumno o una alumna que es víctima repetida de abusos por parte de los compañeros. Cada lámina presenta un tipo de maltrato (físico, verbal y relacional) realizado por una persona o grupo hacia una víctima. El cuestionario SCAN-Bullying está compuesto por un total de 36 ítems divididos en 10 bloques (Caurcel, 2009):

1. Datos del participante.

2. Representación del fenómeno (5 ítems).

3. Maltrato proactivo versus reactivo (1 ítem).

4. Atribuciones causalidad (1 ítem).

5. Caracterización de las víctimas (5 ítems).

6. Experiencia como espectador (1 ítem).

7. Caracterización de los agresores (4 ítems).

8. Emociones morales (13 ítems).

9. Final de la historia (1 ítem).

10. Perfil del participante (5 ítems).

Atendiendo al tipo de respuesta solicitada, el cuestionario SCAN-Bullying (Almeida y Caurcel, 2005) puede considerarse mixto, es decir, que está integrado por algunas preguntas cerradas o semi-cerradas y por otras abiertas. La recogida de información se puede realizar mediante una entrevista semiestructurada o la aplicación de un cuestionario (Almeida, Del Barrio, Marques, Gutiérrez y Van der Meulen, 2001; Almeida y Caurcel 2005; Almeida, Caurcel y Machado, 2006; Almeida, Lisboa y Caurcel, 2007; Del Barrio et al., 2003). 

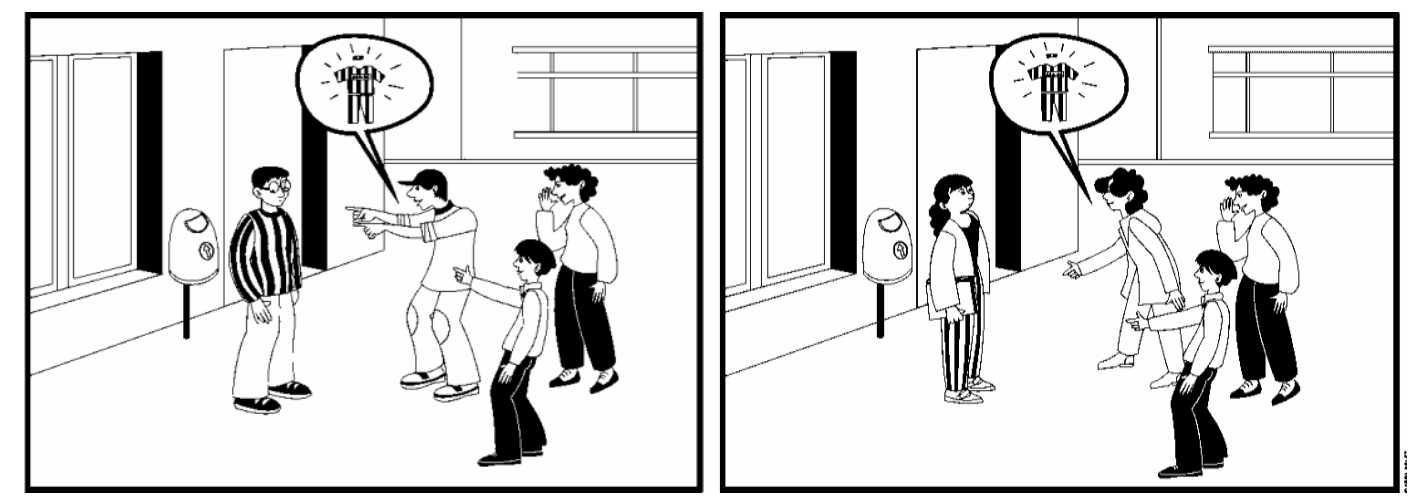

Figura 1. Ejemplos de algunas viñetas del SCAN-Bullying, versión masculina y femenina agresión verbal: un grupo ríe señalando las ropas del alumno/a." (Tomado de Del Barrio, Almeida, et al., 2003, citados en Caurcel 2009, p.56)

\section{Procedimiento}

Tras un contacto inicial con equipo directivo de una escola estadual (escuela administrada por el gobierno del estado federal) situada en la ciudad de Santarém (Pará, Brasil) tuvo lugar una reunión informativa con el cuerpo docente de la escuela para dar a conocer los objetivos del trabajo. Una vez obtenido el acuerdo con la dirección del centro y el cuerpo docente se concertaron los horarios para visitar los cursos. Del mismo modo, y para cumplir con la medida prescrita por el comité de ética de las universidades locales, se envió una carta de información, acuerdo de confidencialidad y solicitud de consentimiento de la realización de la investigación a los padres y tutores del alumnado, de las cuales recibimos debidamente firmadas más de un $90 \%$. Tras las primeras reuniones y con el beneplácito del centro, las familias y las instituciones educativas locales se procedió a la administración de los instrumentos de evaluación.

En primer lugar, y una vez depurados los errores y dificultades encontradas en el estudio piloto, se aplicó la escala DIAS de forma individual para su revisión y cotejo con el alumnado. En una segunda fase, se aplicó colectivamente el instrumento SCAN-Bullying. En este caso, se volvió cuantas veces fue necesario para completar algunos de los ítems que resultaba importante que fueran respondidos por los alumnos. En total se aplicaron 110 cuestionarios de SCAN-BULLYING y se depuraron las 97 escalas de agresión y victimización correspondientes al alumnado participante. 
En el caso de la Escala DIAS, previo al análisis de resultados se procedió a la corrección de cada una de las escalas para determinar qué alumnos fueron nominados como agresores o no agresores. Para este caso se obtuvo el índice asociado a cada uno de los roles obteniendo el promedio del curso y determinando que alumnos estaban por encima o por debajo del mismo para así establecer el alumnado agresor (Machado, 2002). Es importante considerar que para este trabajo sólo se utilizó la puntuación de agresión global, sin trabajar por separado la agresión física, verbal e indirecta, ni la escala de victimización.

Del mismo modo, y en relación con el SCAN-Bullying sólo se analizan las apreciaciones realizadas por los participantes sobre la historia que representan las láminas. En este sentido, se evalúan las atribuciones de ocho pares opuestos de adjetivos: (1) Natural-Aprendido, (2) A propósito-Sin intención, (3) Conflicto-Venganza, (4) Abuso-Juego, (5) Divertido-De maldad, (6) Aceptable-Reprobable, (7) Justo-Injusto, y (8) Correcto-Incorrecto (Almeida y Caurcel, 2005). Los participantes valoraron (escala Likert 1 a 5) el criterio que consideraban oportuno, atribuyendo a cada ítem, un polo de la variable. Así, y tomando como ejemplo el binomio Natural-Aprendido, la caracterización como Natural se correspondería con la valoración 1, y el polo Aprendido con la valoración 5.

\section{Análisis Estadísticos}

Los análisis estadísticos utilizados en el desarrollo de la investigación fueron: la prueba U de Mann-Whitney, análisis de correlación R de Spearman entre las diferentes variables, análisis factorial según variables (r-modal) y según sujetos (q-modal).

Para el cálculo de los coeficientes de correlación y la U de Mann Whitney, se utilizó el software estadísticalStats99 (STA_BAS). Los análisis factoriales fueron calculados a través de un código computacional utilizado por Morrisson (1976) e implementado por Leslie F. Marcus en el leguaje del aplicativo Matlab (Machado 2005). La programación utilizada por Machado (2002) fue re-estructurada para el agrupamiento de factores en los análisis de QModal y R-Modal. 


\section{Resultados}

Los resultados obtenidos tras la administración de la Escala DIAS señalan que el $57,7 \%$ de los participantes es nominado como no agresor (NAGR en adelante) de los cuales el $57,1 \%$ son mujeres y el $42,9 \%$ hombres. Por otro lado, el 42,3\% del alumnado participantes es nominado como agresor (AGR en adelante). En este grupo, un 15,4\% se percibe a sí mismo como agresor. Del grupo nominado como agresor, el $36,6 \%$ son mujeres y el $63,4 \%$ hombres. En relación a las características atribuidas por ambos colectivos -agresores y no agresores-a las situaciones de maltrato representadas en el SCAN-Bullying quedan representadas en el Gráfico 1. El análisis de las respuestas sólo establece diferencias estadísticamente significativas en el polo Aceptable-Reprobable siendo el colectivo de AGR quienes con mayor frecuencia atribuye a la situación el carácter de reprobable ( $\mathrm{U}$ de Mann, $p<, 05 ; \bar{X}_{\mathbf{A G R}}=4,50$; $\left.\bar{X}_{\mathbf{N A G R}}=3,96\right)$. Para el resto de polos atribucionales las diferencias entre ambos grupos fueron pequeñas y no significativas.

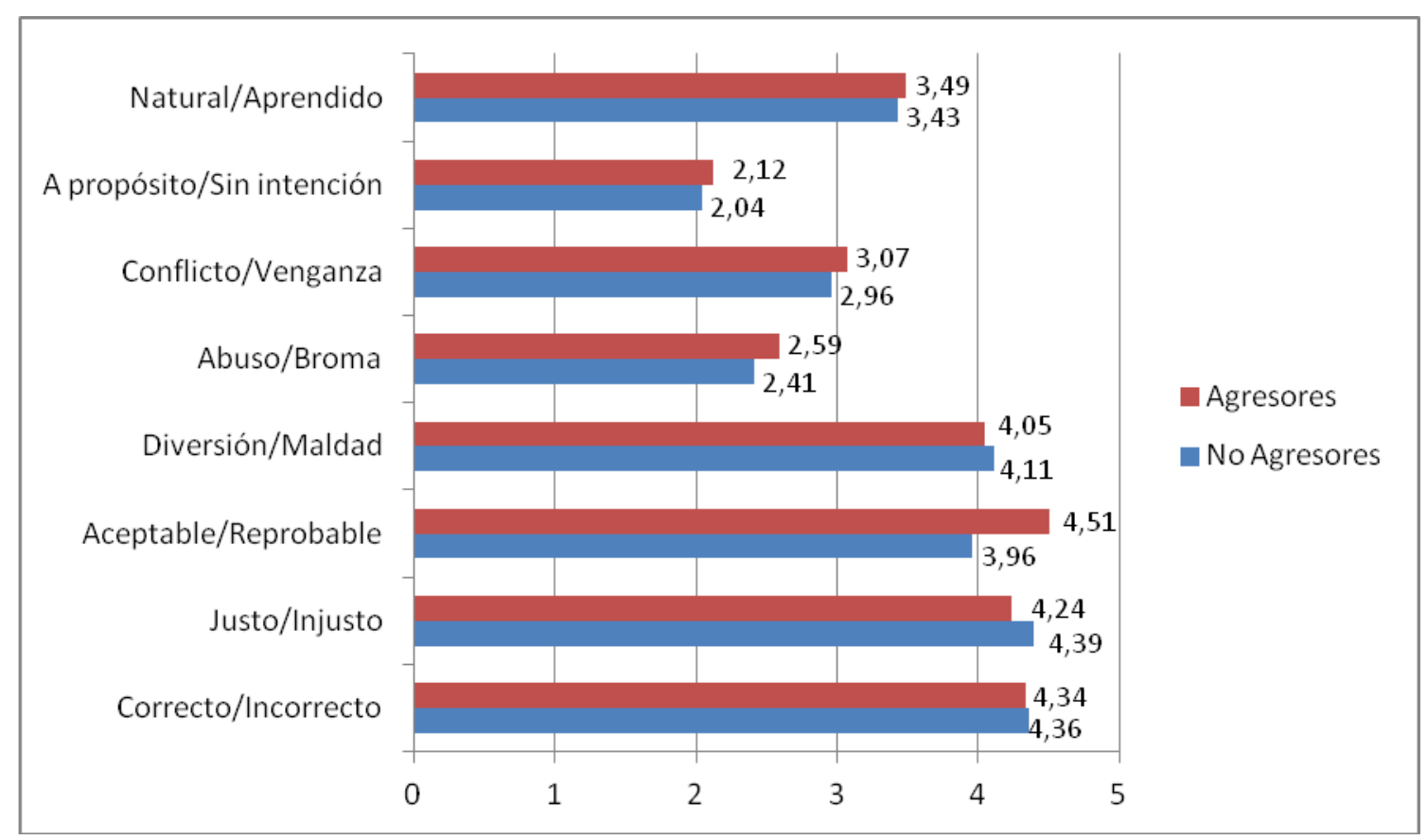

Gráfico 1. Atribuciones a los actos agresivos de la historia según agresores y no agresores 
Grupo nominado como Agresores (AGR)

El análisis correlacional entre las atribuciones del grupo AGR muestra correlaciones positivas entre el polo Justo-Injusto y el polo Correcto-Incorrecto ( $\mathrm{r}=, 70 ; p=, 000)$ correlacionando el polo Justo con el polo Correcto, y el polo Injusto con Incorrecto. Por otra parte, se detectan correlaciones positivas entre los pares Justo-Injusto y el par A propósito-Sin intención $(\mathrm{r}=, 51 ; p=, 000)$. Del mismo modo, se observan correlaciones positivas entre los pares Correcto-Incorrecto y el par A propósito-Sin intención $(\mathrm{r}=, 48 ; p=, 001)$ y correlaciones negativas entre Justo-Injusto y el par Abuso-Juego ( $\mathrm{r}=-, 31 ; p=, 045)$ en el que Justo corresponde a Juego e Injusto a Abuso. Finalmente, aparece una correlación negativa entre el par CorrectoIncorrecto y el par Abuso-Juego $(\mathrm{r}=-, 38 ; p=, 013)$ correcto corresponde a juego e incorrecto a abuso. Entre el resto de pares no se observan correlaciones significativas.

\section{Grupo nominado como no Agresores (NAGR)}

De acuerdo con el análisis correlacional para el grupo NAGR (Tabla 2) se observa una correlación positiva entre el par Natural-Aprendido con el par Conflicto-Venganza (r=,37; $p=, 005)$ correspondiéndose la atribución de Natural con Conflicto y el polo Aprendido con Venganza.

Del mismo modo, el análisis correlacional permite establecer varias correlaciones negativas entre los pares atribucionales. En primer lugar, se observa que el par NaturalAprendido correlaciona negativamente con: A propósito-Sin intención (r=-,41; $p=, 001)$ siendo la correspondencia del polo natural con sin intención y del aprendido con a propósito; Abuso-Juego ( $\mathrm{r}=-, 37 ; p=, 004)$ donde natural corresponde a juego y aprendido a abuso; Aceptable-Reprobable $(\mathrm{r}=-, 50 ; p=, 000)$ en la que natural corresponde con aceptable y aprendido con reprobable; Justo-Injusto $(\mathrm{r}=-, 29 ; p=, 02)$ en el que natural corresponde con justo y aprendido con injusto.

Del mismo modo, el análisis realizado también muestra correlaciones negativas entre el par Conflicto-Venganza y otros polos atribucionales tales como: Abuso-Juego (r=-,27; $p=, 000)$ en la que conflicto corresponde con juego y venganza con abuso; AceptableReprobable $(\mathrm{r}=-, 33 ; p=, 012)$ en el que conflicto se corresponde con juego y venganza con 
abuso; y, Justo-Injusto $(\mathrm{r}=-, 29 ; p=, 029)$ con la correspondencia de conflicto a justo y venganza a injusto.

Tabla 2. Correlaciones entre las diferentes variables y grupos AGR-NAGR

\begin{tabular}{|c|c|c|c|c|c|c|c|c|}
\hline & & $\begin{array}{l}\text { Propósito } \\
\text { Sin inten- } \\
\text { ción }\end{array}$ & $\begin{array}{l}\text { Conflicto } \\
\text { Venganz }\end{array}$ & $\begin{array}{c}\text { Abuso } \\
\text { Juego }\end{array}$ & $\begin{array}{c}\text { Diversión } \\
\text { Maldad }\end{array}$ & $\begin{array}{l}\text { Aceptable } \\
\text { Reproba- } \\
\text { ble }\end{array}$ & $\begin{array}{l}\text { Justo } \\
\text { Injus- } \\
\text { to }\end{array}$ & $\begin{array}{l}\text { Correcto } \\
\text { Incorrec- } \\
\text { to }\end{array}$ \\
\hline Natural & AGR & - & - & - & - & - & - & - \\
\hline Aprendido & NAGR &,- 41 & ,37 &,- 37 & - &,- 50 &,- 29 & - \\
\hline Propósito & AGR & - & - & - & - & - &, 51 & ,48 \\
\hline Sin Inten. & NAGR & & - & - & - & - & - & - \\
\hline Conflicto & AGR & & & - & - & - & - & - \\
\hline Venganza & NAGR & & &,- 27 & - &,- 33 &,- 29 & - \\
\hline Abuso & AGR & & & & - & - &,- 31 &,- 38 \\
\hline Juego & NAGR & & & & - & - & - & - \\
\hline Diversión & AGR & & & & & - & - & - \\
\hline Maldad & NAGR & & & & & ,40 & - & - \\
\hline Aceptable & AGR & & & & & & - & - \\
\hline $\begin{array}{l}\text { Reproba- } \\
\text { ble }\end{array}$ & NAGR & & & & & & ,43 & - \\
\hline Justo & AGR & & & & & & & ,70 \\
\hline Injusto & NAGR & & & & & & & - \\
\hline
\end{tabular}

Análisis factorial: R-Modal y Q-Modal

Con el análisis factorial se persiguen dos objetivos. En primer lugar, reducir la complejidad del análisis reduciendo un número elevado de variables independientes a un número inferior de factores que son variables abstractas agregadas (análisis R-modal). Y en segundo lugar, conformar grupos de sujetos con características similares utilizando dichos factores (análisis Q-modal). En este caso, el interés específico del análisis q-modal está en detectar si los diferentes grupos de sujetos que se distinguen a través de estos factores relacionados con la apreciación de actitudes y acciones, están compuestos por individuos clasificados como agresores o como no agresores, según la escala DIAS.

Al realizar el análisis factorial se tiene en cuenta el análisis de ji-quadrado $\left(\chi^{2}{ }_{\mathrm{k}}\right)$ para la distribución y así poder escoger mejor el número de factores (Machado 2005). En el Gráfico 2 se observa el movimiento de la información acumulada. 

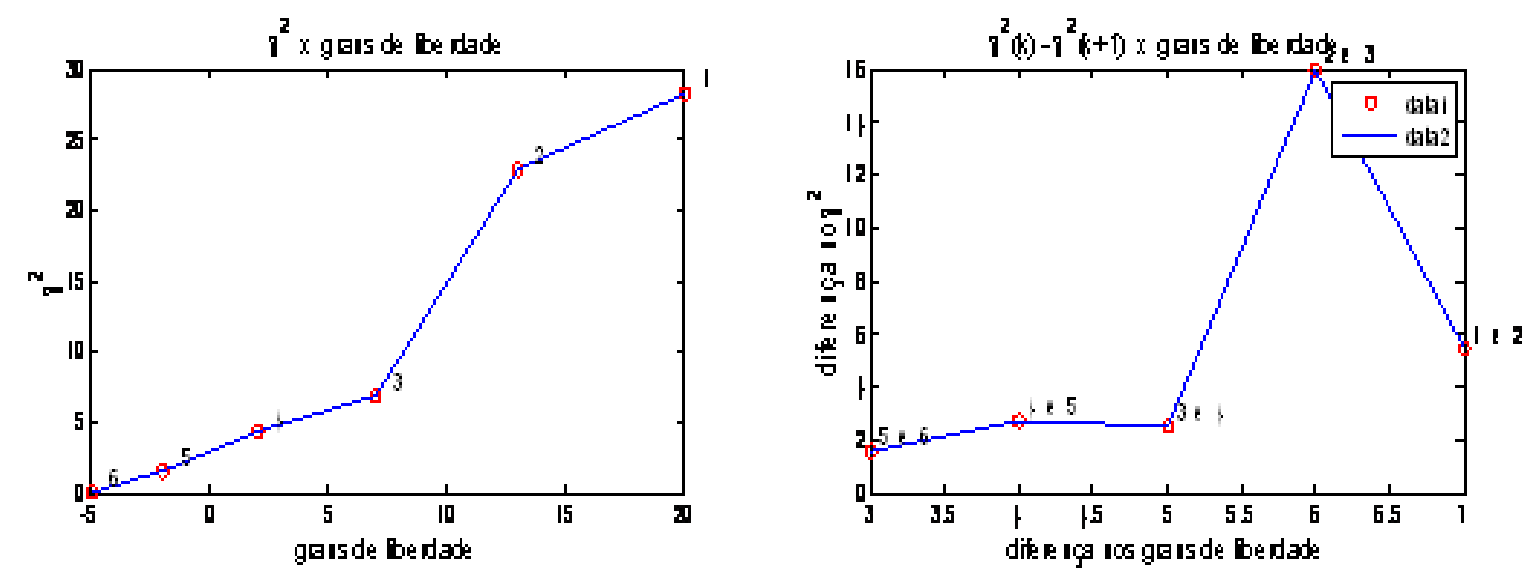

Gráfico 2. Variación del ji-quadrado, grado de libertad y diferencias.

Para el grupo AGR, el factor 1 representa al conjunto de las variables predominantes: par 1 Diversión-Maldad, par 7 Justo-Injusto y par 8 Correcto-Incorrecto. Tanto para el factor 2 como para el factor 3, el par 2 A propósito-Sin intención presenta mayor relevancia. Los tres factores juntos representan el $49 \%$ de la variabilidad del conjunto de las variables (Gráfico 3).
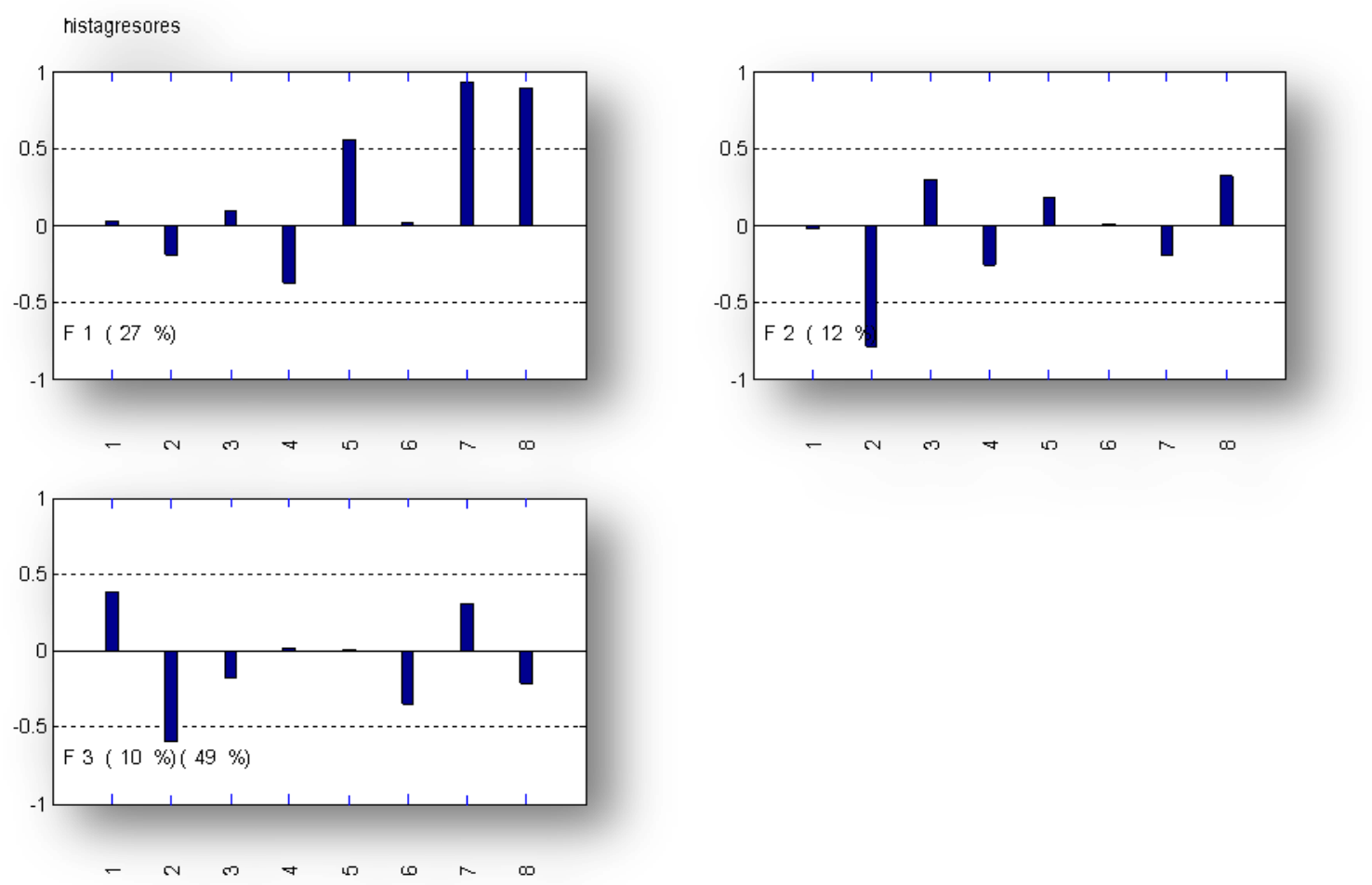

Gráfico 3. Percepción del grupo AGR sobre lo que sucede en la historia 
Por último, analizando la percepción del grupo NAGR y los tres factores que representan un 51\% de la variabilidad del conjunto de las variables encontramos los siguientes resultados. El factor 1 representa el conjunto de las variables: par 2 A propósito-Sin intención, par 5 Divertido-De maldad y par 7 Justo-Injusto. En el factor 2, sólo la variable conflicto-venganza tuvo relevancia y, en el factor 3, solamente la variable correcto-incorrecto (Gráfico 4).
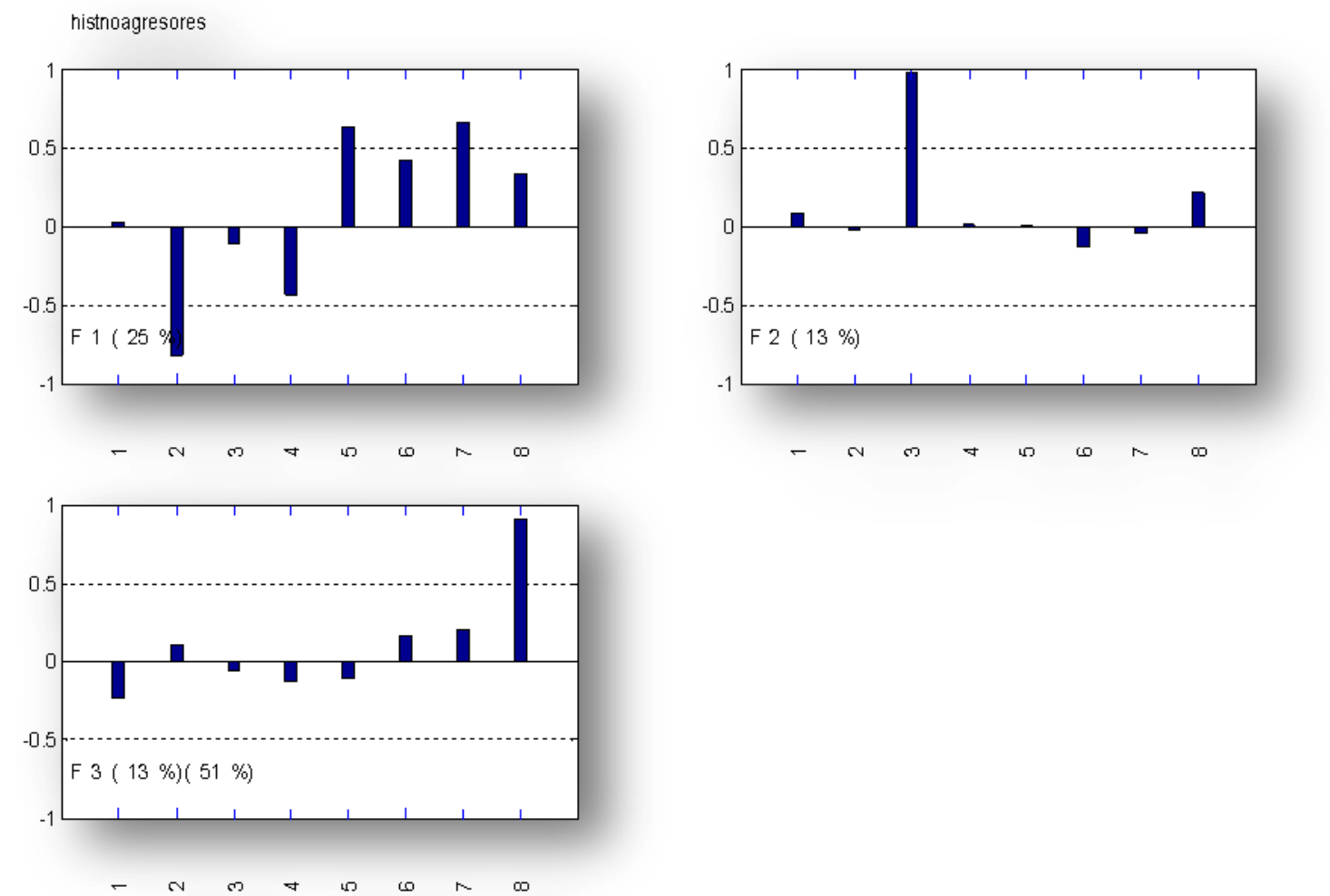

Gráfico 4. Percepción del grupo NAGR sobre lo que sucede en la historia.

En cuanto análisis Q-modal, busca encontrar las interrelaciones existentes entre los elementos de un determinado conjunto representativo con base en una definición de similitud entre los sujetos (Machado, 2005). Se observa (Gráfico 5) la agrupación específica de grupos de alumnos nominados como AGR y NAGR, además de otro tipo de agrupaciones. En total se establecen 4 grupos importantes (Gráficos 5 y 6) si bien aparecen otros grupos aunque muy dispersos y mezclados en su formación entre alumnos nominados AGR y NAGR:

- Grupo 1 ( $\left.\frac{M}{7}\right)(90,77,65,78,80,54,81,87,70,93,86,84,85,73,92)$ : con un total de 16 sujetos y con su centro marcando un valor de 0,2. Este grupo está formado exclusivamente por alumnos nominados NAGR, presentando valores medios-altos en el factor 1, altos en el factor 2 y medios en el factor 3. 
- Grupo $2(\triangle)(95,56,52,97,66,83,75,76)$ : con un total de 9 sujetos y con su centro marcando un valor de 2. En este caso, también está formado exclusivamente por alumnos nominados NAGR. Presenta valores medios en el factor $1 \mathrm{y}$ mayor variabilidad, valores medios en el factor 2, y, valores medios-bajos en el factor 3.

- Grupo 3 (*) $(79,89,68)$ : consta de tres miembros. Este grupo ubicado en el centro, presenta valores relativamente bajos en el factor 1, medios en el factor 2 , y altos en el factor 3. Al igual que en los casos anteriores, también está formado exclusivamente por alumnos nombrados NAGR.

- Grupo 4 ( $\square$ ) (38-7-31-17-5-10-27-14-26-34-8): formado por 11 miembros nominados como AGR. El grupo presenta valores medios-altos en el factor 1, medios en el factor 2 , y, altos en el factor 3 .

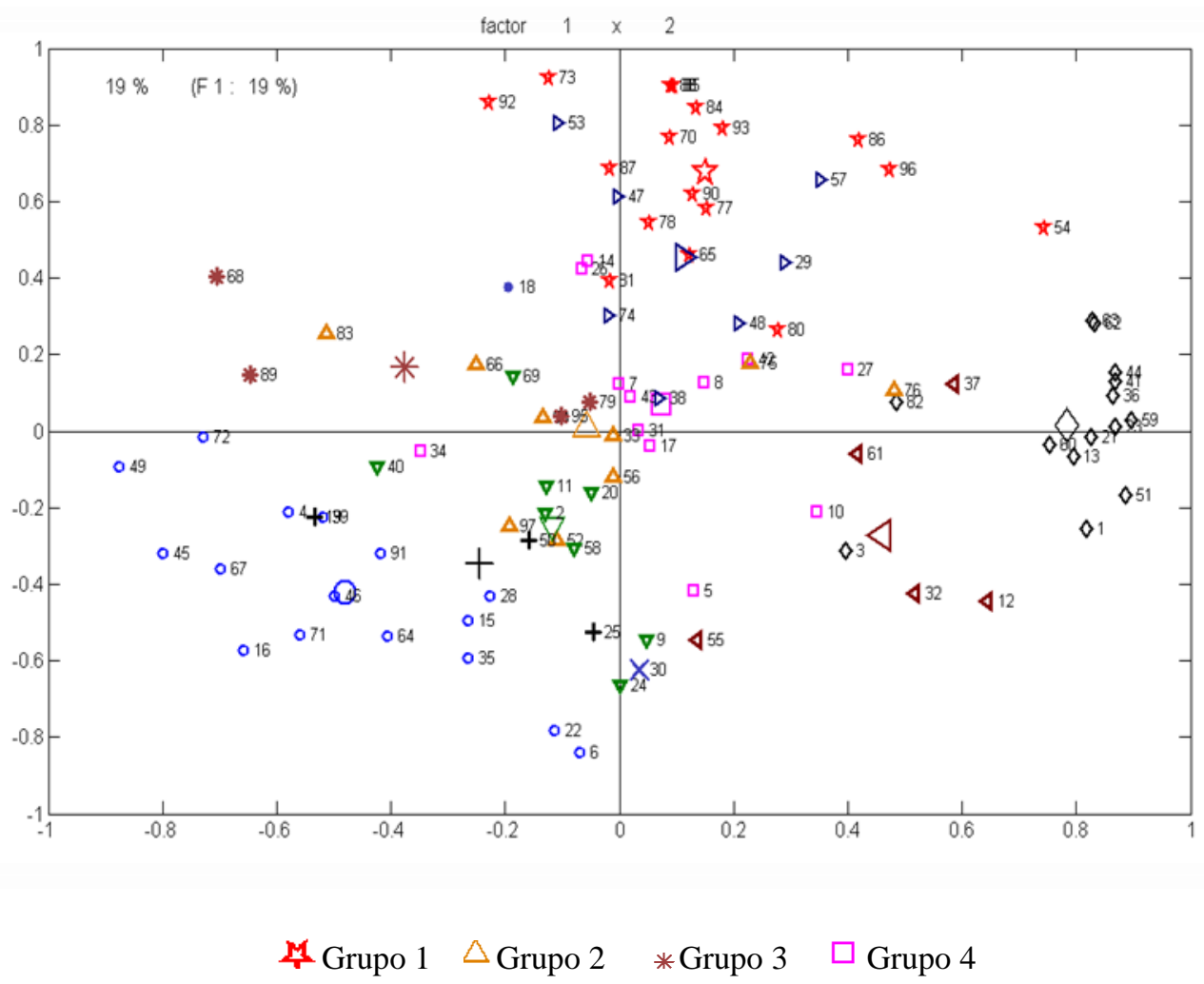

Gráfico 5. Cargas factoriales de los dos factores que cargan los mayores valores de información (factores 1 y 2). 

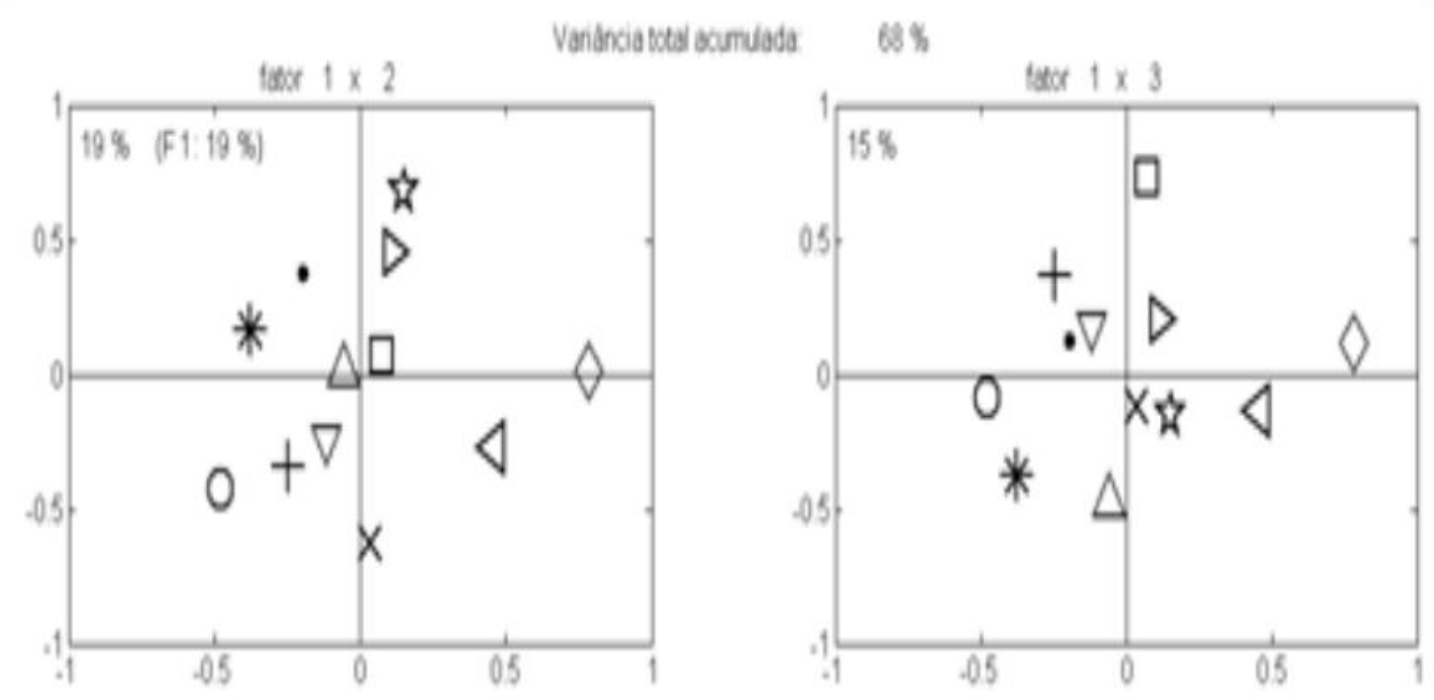

Gráfico 6. Cargas factoriales de los centroides de los tres factores que cargan los mayores valores de información (factores 1,2 y 3).

\section{Discusión y conclusiones}

La proporción de agresores $(42,3 \%)$ encontrada en el estudio parece ser, a primera vista, más alta que en otras investigaciones sobre la temática. Avilés (2006) informa que el $11,6 \%$, del alumnado dice haber estado implicado en situaciones de maltrato a lo largo del trimestre de forma sistemática, bien como agresor $(5,9 \%)$ o como víctima $(5,7 \%)$. De acuerdo a la descripción de Benítez y Justicia (2006), mencionando a Olweus, el 17,6\% del alumnado señaló haberse visto involucrado en alguna situación de maltrato, bien como agresor (7\%), bien como víctima (9\%), Sin embargo, si observamos el porcentaje de alumnado en la presente investigación que se percibe a sí mismo como agresor el porcentaje cae hasta un 15,4\% siendo tal porcentaje similar a los encontrados en estudios precedentes (Avilés, 2006; Benítez y Justicia 2006; Caurcel, 2009; García y Madriaza, 2006; Informe del Defensor del Pueblo, 2007; Rigby, 1999).

La alta incidencia de agresores registrada en el presente trabajo y las correlaciones, en parte bastante estrechas, establecidas entre los diferentes índices de agresión coinciden con las encontradas en trabajos previos (Gutiérrez, 2007). En este sentido, se pone en evidencia la 
presencia de una proporción relativamente alta de agresores reactivos caracterizados por la activación emocional del enfado o miedo como respuesta a altos niveles de estrés (Andreu, Peña y Ramírez, 2009; Caurcel, 2009; Chaux 2005; Cerezo, 2009; Duran 2004). No obstante, la hipótesis planteada en el estudio no se ratifica en su totalidad dado que el alumnado AGR comparte, con el alumnado NAGR, sentimientos y actitudes negativos frente al maltrato si bien los primeros la ven como justificable como respuesta a una hostilidad previa. Esto último, coincide con el perfil de agresor reactivo y no con el esperado perfil de agresor proactivo.

En general, las valoraciones de los nominados AGR y los nominados NAGR son similares. En promedio, los participantes tienden a considerar que los actos representados en el $S C A N-B u l l y i n g$ son hechos de maldad, reprobables, injustos e incorrectos y que caracterizan como abusos. En cuanto a la variable Natural-Aprendido, las valoraciones de ambos grupos quedan en un término medio. En relación con la variable Conflicto-Venganza, para el grupo AGR queda en un término intermedio, mientras que entre los miembros del NAGR se considera que el maltrato es debido más a conflictos momentáneos que a actos de venganza. Las correlaciones existentes entre las variables analizadas y el agrupamiento factorial de las mismas, indican que los valores y las actitudes entre el alumnado (AGR y NAGR) hacia el maltrato entre iguales son consistentes, positivos y socialmente correctos.

Un dato interesante surge cuando observamos que es el grupo de AGR quienes de firna significativa, con mayor frecuencia que el grupo de NAGR, señalan el maltrato como un hecho reprobable. Además, los agresores son conscientes de la injusticia y de la incorrección del maltrato, a pesar de que ellos mismos maltratan a los compañeros de forma frecuente.

Sin embargo, y pesar de la frecuencia con la que maltratan a los compañeros, el grupo de los agresores tiene dificultades para señalar si el maltrato se debe a conflictos entre ellos o actos premeditados de venganza. Si consideramos las dos ideas señaladas, la frecuencia alta de ocurrencia y la dificultad para idetificar el origen del problema, el maltrato aparece como algo inherente a la forma de interaccionar de los miembros de este grupo. En este sentido, cabe señalar que los agresores reactivos perciben hostilidades frente a situaciones ambigüas. La percepción de hostilidad provoca reacciones agresivas, justificadas para el sujeto, que generan a su vez hostilidades dando lugar a un clima general de agresividad recíproca (Dodge y Crick, 1996). En esta línea, y si bien los AGR son más tajantes en la reprobación de los actos de abuso, es interesante observar como el alumnado agresor afirma que si existe abuso es jus- 
to comportarse de la misma forma. De igual forma, consideran que si hay abuso es correcto defenderse de la misma forma. Del mismo modo, se puede observar como los AGR a pesar de reconocer la injusticia y la incorrección del maltrato señalan también la falta de intencionalidad del mismo. En cada una de estas correlaciones se pueden interpretar los sesgos y los procesos de apreciación en lo referente a lo que los participantes determinan como hostil o no hostil. Cuando las intenciones de la otra persona se perciben como hostiles se incrementa el conflicto y se justifica el responder de la misma forma aunque tal situación sea reprobable (Avilés, 2006; Dodge, 1986).

El anális factorial R-modal y Q-modal apoya las conclusiones anteriores. Por una parte, el grupo de NAGR (Grupo 1) señala la maldad, la injusticia y la intencionalidad presentes en el maltrato y aunque lo asocian a situaciones derivadas de conflictos no lo consideran correcto. Es razonable suponer, y en relación a lo visto anteriormente, que esta apreciación se corresponde con actitudes que no están cargadas de rencor y rabia, sino más propensas a resolver los conflictos que surgen de forma positiva. Por otra parte, el grupo sólo por AGR (Grupo 4) afirma que el maltrato es incorrecto, injusto y reprobable, aunque no considera que sea un comportamiento intencionado y que es justo maltratar si te maltratan. La respuesta agresiva se asocia tanto a situaciones conflictivas entre el alumnado como a actos de represalia.

En el juego de la interacción humana surgen conflictos que ayudan al ser humano a desenvolver habilidades sociales. Sin embargo, cuando los conflictos no se encaran adecuadamente generan una agresividad que se convierte en violencia si no funcionan los elementos mediadores para solucionar el conflicto (Justicia, Arco y Benítez, 2003). En el presente estudio, el alumnado participante tiene una percepción clara del problema y no tienen problemas en identificar las diferentes situaciones como actos de maltrato tal y como acontece en estudios previos (Almeida y Caurcel, 2005; Almeida et al., 2001, 2003; Del Barrio et al., 2003). Del mismo modo, caracterizan el maltrato como un acto reprobable, incorrecto y de maldad lo que conlleva la asunción de atribuciones sociales adecuadas. No obstante, debe considerarse que los agresores tienden a justificar la práctica de tales comportamientos como respuesta ante situaciones hostiles. En términos de intervención será interesante potenciar el rechazo hacia el maltrato entre iguales a la vez que eliminamos actitudes que puedan justificar su existencia.

Los resultados obtenidos deben acogerse con cautela debido la cantidad de participantes en el estudio y al carácter localista de la muestra. Del mismo modo, sería interesante en el 
futuro comparar los datos del presente estudio con los resultados de la escala de victimización $\mathrm{y}$, en ese sentido, agrupar cuatro grupos; los nominados agresores, los nominados victimas, los agresores-victimas y los que no asumen ningún rol. De esta forma podrían obtenerse otros elementos importantes que podrían mediar en el abordaje de una convivencia basada en valores auténticos que promuevan la paz, la solidaridad y la aceptación del otro.

\section{Referencias}

Abramovay, M. y Rua, M. (2002). Drogas e violência nas escolas. Brasília: UNESCO

Almeida, A. y Caurcel, M. (2005). Questionário Scan-Bullying. Versão Portuguesa e Castelhana. Registro de obra na Inspecção-Geral das Actividades Culturais, Ministério da Cultura.

Almeida, A., Caurcel, M. J. y Machado, J. C. (2006). Perceived characteristics of victims, according to their victimized peers. Electronic Journal of Research in Educational Psychology, 9, 4(2), 371-396

Almeida, A., Del Barrio, C., Marques, M., Fernández, I., y Gutiérrez, H. (2001). A scriptcartoon narrative of peer-bullying in childrenand adolescents. In M. Martínez (Ed.), Prevention and control of aggression and its impact on its victims (161-168). New York: Kluwer Academic/ Plenum

Almeida, A., Lisboa, C. y Caurcel, M. J. (2007). ¿Por qué Ocurren los Malos Tratos entre Iguales? Explicaciones Causales de Adolescentes Portugueses y Brasileños. Revista Interamericana de Psicología/Interamerican Journal of Psychology, 41(2), 107-118.

Andreu, J. M., Peña, M. E., y Ramírez, J. M. (2009). Cuestionario de Agresión Reactiva y Proactiva: Un instrumento de medida de la agresión en adolescentes. Revista de Psicopatología y Psicología Clínica, 14(2), 37-49

Avilés, J. M. (2001). Bullying: intimidación y maltrato entre el alumnado. Bilbao: Stee-Eilas

Avilés, J. M. (2006). Diferencias de atribución causal en el bullying, entre sus protagonistas. Electronic Journal of Research in Educational Psychology, 9, 4(2), 201-220

Bandura, A. (1973). A social learning analysis. Englewood Cliffs: Prentice Hall 
Bandura, A. (1983). Psychological mechanisms of aggression. En R. G. Green y E. I. Donnerstein (Eds.), Aggression, theoretical and empirical views (pp. 1-40). New York: Academic Press

Benítez, J. L. y Justicia, F. (2006). El Maltrato entre iguales: descripción y análisis del fenómeno. Electronic Journal of Research in Educational Psychology, 9, 4(2), 151-170.

Benítez, J. L., Almeida, A. y Justicia, F. (2005). Educación para la convivencia en contextos escolares: una propuesta de intervención contra los malos tratos entre iguales. Apuntes de Psicología, 23(1), 27-40.

Berkowitz, L. (1963). Aggression: a social learning analysis. New York: Mc Graw Hill

Berkowitz, L. (1993). Aggression: its causes, consequences, and control. New York: McGraw Hill.

Björkqvist, K., Lagerspetz, K. y Österman K (1992). The Direct \& Indirect Aggression Scales. Åbo Akademi University, Department of Social Sciences, Vasa, Finland.

Caurcel, M. J. (2009). Estudio Evolutivo del maltrato entre iguales desde la percepción y el razonamiento moral de los implicados. Tesis doctoral. Universidad de Granada, España.

Chaux, E. (2005). El programa de prevención de Montreal: Lecciones para Colombia. Revista de Estudios Sociales, 21, 11-25.

Collel, J. y Escudé, C. (2004). Rol de les emocions en els processos de maltractament entre alumnes. Ámbits de Psicopedagogía, 12, 21-26.

Cowie, H. (2000). Bystanding or standing by: gender issues in coping with bullying in English schools. Aggressive Behavior, 26, 85-97

Crick, N. R. y Dodge, K. A. (1994). A review and reformulation of social information processing mechanisms in children's social adjustment. Psychological Bulletin, 115, 74-101.

Del Barrio, C., Almeida, A. y K., van der Meulen, K., Barrios, A. y Gutiérrez, H. (2003). Representaciones acerca del maltrato entre iguales, atribuciones emocionales y percepción de estrategias de cambio a partir de un instrumento narrativo: SCAN-Bullying. Infancia y Aprendizaje, 26(1), 63 - 78

Del Barrio, C., Gutiérrez, H., Barrios, A., Van der Meulen, K. y Granizo, L. (2005). Maltrato por abuso de poder entre escolares, ¿de qué estamos hablando? Revista de Pediatría y Atención Primaria, 7, 75-100. 
Devine, J. (2002). A mercantilização da violência escolar. En E. Debarbieux y C. Blaya (Eds.), Violência nas escolas e políticas públicas (207-223). Brasilia: UNESCO (original inglés: Violence in School and Public Policies, Paris: Elsevier)

Dodge, K. A. (1986). Social information processing variables in the development of aggression and altruism in children. En C. Zahn-Waxlet, E. M. Cummings, y R. Iannotti (Coords.), Altruism and aggression. Cambridge: Cambridge University Press

Dodge, K. A. y Coie, J. D. (1987). Social information processing factors in reactive and proactive aggression. Child Development, 51, $162-170$

Dodge, K. A. y Crick, N. R. (1996). Social information-processing mechanisms in reactive and proactive aggression. Child Development, 67, 993-1002.

Durán, A. (2004). La agresión escolar en los centros de segundo ciclo de ESO de Granada capital y su provincia. Tesis doctoral no publicada. Universidad de Granada.

Fante, C. (2005). Fenômeno Bullying. Como prevenir a violência nas escolas e educar para a paz. Campinas, SP (Brasil): Verus Editora.

Fernández, M. (2006). Representaciones mentales, atribuciones causales y estados emocionales de adolescentes entre 11 y 15 años víctimas de maltrato entre iguales. Proyecto de Investigación Tutelada. Universidad de Granada. España.

Garaigordobil, M. y Oñederra, J. A (2008). Estudios epidemiológicos sobre la incidencia del acoso escolar e implicaciones educativas. Informació Psicológica, 94, 14-35.

García, M. y Madriaza, P. (2005). Estudio cualitativo de los determinantes de la violencia escolar en Chile. México: Artigos.

Gonçalves, L. A. y Spósito, M. (2002). Iniciativas públicas de redução da violência escolar no Brasil. Cadernos de pesquisa, 115, 101-138.

Gutiérrez, R. (2007). Sentimientos y emociones de víctimas y agresores. Tesina Doctoral no publicada. Universidad de Granada.

Informe de Defensor del Pueblo (2007). Violencia Escolar: el maltrato entre iguales en la Educación Secundaria Obligatoria 1999-2006. Recuperado el 15/11/2007, de http://www.defensordelpueblo.es/documentacion/informesmonograficos/ViolenciaEsco lar2006.pdf

Jimersen, S., Swearer, S. y Espelage, D. (2010). Handbook of Bullying in Schools: an international perspective. Nueva York: Routledge.

Justicia, F., Arco, J. L. y Benítez, J. L. (2003). El fenómeno de la violencia escolar: que és y como intervenir. En Herrera, H.; Lorenzo, O.; Mesa, M. y Alemany, Y. (Coords.) In- 
tervención Psicoeducativa: una perspectiva multidisciplinar. Granada. Grupo Editorial Universitario.

Machado, C. J. F. (2005). Modelagem geoquímica e de Fluxos dos Sistema Aquifero subterrãneo na bacia sedimentar do Araripe. Tese doutoral, Fortaleza-CE, Brasil.

Machado, M. F. C. de. (2002). A Vitimação entre Pares em Contexto Escolar - Um estudo das percepções de solidão, auto-conceito e maus tratos de crianças vitimadas. Tese de Mestrado, Braga, Universidade do Minho.

McLoughlin, C. (2009). Positive Peer Group Interventions: An Alternative to Individualized Interventions for Promoting Prosocial Behavior in Potentially Disaffected Youth. Electronic Journal of Research in Educational Psychology, 7(3), 1131-1156.

Moos, R. H., Moos, B. S. y Tricket, E. J. (1988). Escalas de Clima social. Madrid: TEA Ediciones.

Morrison, D. F. (1976). Multivariate Statistical Methods (2 $2^{\text {nd }}$ ed.). New York: McGraw Hill.

Olweus, D. (1991). Bully/victim problems among schoolchildren: Basic facts and effects of a school based intervention program. En D. J. Pepler y K. H. Rubin (Eds.), The development and treatment of childhood aggression (411-448). Hillsdale, NJ: Erlbaum.

Olweus, D. (1993). Bullying at school: What we know and what we can do. Oxford. UK: Blackwell.

Ortega R. y Merchán. J. (1997). Agresividad y violencia. El problema de la victimización entre escolares. Revista de Educación, 313, 7-28

Phillips, V. y Dewey, G. (2012). Identifying Victims of Bullying: Use of Counselor Interviews to Confirm Peer Nominations. Professional School Counseling, 15(3), 123-131.

Ramírez, S. y Justicia, F. (2006). El maltrato entre escolares y otras conductas problema para la convivencia. Electronic Journal of Research in Educational Psychology, 9, 4(2), 265-289.

Rigby, K. (1999). Peer victimization at school and the health of secondary students. British Journal of Educational Psychology, 69, 95-104.

Smorti, A. (1994). II pensiero narrativo. Construzione di storie e sviluppo della conoscenza sociale. Firenze: Giunti

Thum, J. (2003). Educação dos sentimentos. Canoas, Brasil: Ed. ULBRA

Turnbull, J. y Patterson, B. (1999). Aggression and violence: approaches to effective management. London: Macmillan. 
Rosaura Gutiérrez et al.

Yuen Man Yan, Y. (2007). Multidimensional self-concept of proactively aggressive adolescents and reactively aggressive adolescents in Hong Kong. Hong Kong: City University. 